ORIGINAL ARTICLE

\title{
Detection of hypertension in the emergency department
}

\author{
J Fleming, C Meredith, J Henry
}

Emerg Med J 2005;22:636-639. doi: 10.1136/emj.2004.015040

See end of article for authors' affiliations

.....................

Correspondence to:

Mr J Fleming, Academic

Department of Accident

and Emergency Medicine,

Imperial College, St Mary's

Hospital, London W2

INY, UK; john.fleming@

imperial.ac.uk

Accepted for publication 16 December 2004

\begin{abstract}
Objectives: To assess whether an emergency department (ED) is a suitable location for the targeted screening of hypertension.

Methods: This was a prospective targeted screening study based at the ED of an inner city teaching hospital. Non-acute subjects over 18 years were recruited consecutively from the "minors" section of the $E D$ and invited to participate. All subjects had their blood pressure measured twice. A verbal numerical pain score (PS) out of 10 using a visual analogue scale was obtained. Those with a mean systolic blood pressure $>140 \mathrm{mmHg}$ or a mean diastolic blood pressure $>90 \mathrm{mmHg}$ (WHO JNC stage 1 hypertension) were invited for a subsequent follow up measurement. The primary outcome measure was the proportion of subjects with hypertension at follow up. The secondary outcome measure was the correlation between a subject's mid blood pressure (MBP) and their PS.

Results: In total, 765 subjects were tested, of whom 213 subjects were hypertensive at presentation (28.7\%). After excluding those on anti-hypertensive medication $(n=43 ; 5.6 \%)$ and those who were nonUK residents ( $n=44 ; 5.8 \%), 126$ subjects were invited for follow up, of whom 51 subjects actually attended ( $40 \%$ attendance, $6.6 \%$ of study population). The MBP of those who re-attended was significantly lower than at presentation $(\mathrm{p}<0.001) ; 39$ subjects $(5 \%$ of the study population, $76.4 \%$ of those attending follow up) remained hypertensive. There was no correlation between a subject's PS and their MBP (Pearson correlation coefficient $=-0.02$ ). A $10 / 10$ PS was associated with an $8.4 \mathrm{mmHg}$ rise in MBP compared to the mean MBP of subjects with PS $0-9(p<0.1)$. Of those originally presenting with PS $>5 / 10,62 \%$ still had hypertension at follow up when the painful stimulus was significantly reduced (mean PS $=0.6$ ).

Conclusion: The ED provides an opportunity for identifying those individuals with hypertension who may otherwise remain undiagnosed. Caution is advised when diagnosing hypertension in those individuals suffering from anxiety and/or acute severe pain on presentation.
\end{abstract}

$\mathrm{H}$ ypertension is a major risk factor for cardiovascular diseases, ${ }^{1}$ which are still the leading cause of death in Europe and North America. ${ }^{2}$ In the UK, approximately $40 \%$ of all strokes are attributable to systolic blood pressures $\geqslant 140 \mathrm{mmHg}$. In the general population, $37 \%$ of coronary heart disease in men and $27 \%$ in women is attributable to hypertension. ${ }^{3}$ In addition, a recent meta-analysis of individual participant data from separate prospective studies has confirmed that blood pressure is strongly related to vascular and overall mortality, without any evidence of a threshold down to at least $115 / 75 \mathrm{mmHg}{ }^{4}$

A recent National Heart Forum report highlighted the "major social class, regional and ethnic differences in rates of heart disease and risk factors". For example, the declining rates of heart disease appear to be concentrated among nonmanual social groups. ${ }^{5}$ In addition, the urban poor have little access to health care delivery sites other than an emergency department (ED), and regard it as their regular and frequently their only source of care. ${ }^{6}$ The identification of undiagnosed hypertension in the community would serve as a powerful tool in combating heart disease. The UK National Service Framework aims to reduce variations in morbidity and mortality and to improve the overall standard of care for cardiac patients. ${ }^{7}$

Each year, about 15 million people attend EDs throughout the UK. ${ }^{8}$ This represents approximately $25 \%$ of the population. Depending on the location of the department (rural or urban; inner city or suburban), a proportion of these individuals, ranging from approximately 10 to $25 \%$ are not registered with a general practitioner (GP). For these patients, opportunistic screening for numerous potentially life threatening conditions is not available. We set up a small study to find out whether targeted screening for hypertension was feasible in the ED, an environment in which a largely unselected and often disadvantaged population can be examined and screened for disease.

\section{METHODS}

\section{Design and outcome measure}

This was a prospective targeted screening study. The primary outcome measure was the proportion of subjects with sustained hypertension at follow up after measurement at their initial ED presentation. The systolic and diastolic data obtained for each subject were used to calculate their mid blood pressure (MBP). The MBP (half the systolic blood pressure (SBP) plus half the diastolic blood pressure (DBP)) has recently been shown to confer the highest degree of predictability for stroke and coronary heart disease risk. ${ }^{4}$ A mean of two MBP measurements on the first sitting was compared to the MBP from the last of three measurements taken at follow up. The mean of two MBP measurements at presentation was used because any future ED based hypertension screening programme would be unlikely to measure BP more than twice on presentation. The third reading was used at follow up as this was felt to be a better indicator of a subject's resting BP and may have alleviated any haemodynamic effects caused by stress and anxiety.

Abbreviations: BHS, British Hypertension Society; DBP, diastolic blood pressure; ED, emergency department; GP, general practitioner; $M B P$, mid blood pressure; PS, pain score; SBP, systolic blood pressure 
The secondary outcome measure was the correlation between a subject's MBP and their pain score (PS).

\section{Location and materials}

Researcher shifts normally lasted for 3 hours, with each 24 hour period fully covered during the length of the study. The same researcher, a fourth year medical student competent in measuring blood pressure by auscultation, performed all the blood pressure measurements in order to reduce interobserver variability and terminal digit preference, and to and give consistency to any error of measurement. ${ }^{9}$ A single mercury sphygmomanometer was used for all measurements and was calibrated and tested according to British Hypertension Society (BHS) guidelines. This was used in preference to an automated device because few studies have assessed the validity of these devices and up to $24 \%$ have demonstrated clinically significant errors. ${ }^{10}$ Automated BP devices can overestimate BP in hypotensive patients, and manual measurement can be more reliable in a trauma situation. ${ }^{11}$

\section{Subjects}

Subjects were selected consecutively during the shifts. Each potential subject was given a verbal explanation and written consent was obtained. All patients invited to participate in the study were in triage categories 3-5 (non-urgent). Patients aged $<18$ years were excluded from the study as were those with suspected right upper arm or right shoulder injuries.

\section{Pain scores}

A visual analogue pain score sheet was constructed using several languages to help pain assessment, with scores out of $10 .{ }^{12}$

\section{Measurement}

The study adhered to strict BHS protocol. Manometer position and the position and height of each subject's arm remained constant throughout. After a systolic palpatory estimation of systolic pressure was performed, auscultatory blood pressure was measured using a $26 \times 12 \mathrm{~cm}$ cuff. A $40 \times 12 \mathrm{~cm}$ cuff was used only when the cuff did not hold during inflation. The diastolic reading was noted at Korotkoff phase V, except in pregnant women, when Korotkoff phase IV was used. A rest period of 5 minutes was observed before the first measurement was performed. All measurements were made on the right arm in the seated position. A second reading was obtained 2 minutes after the first.

\section{Follow up}

Those subjects who either had a mean SBP $\geqslant 140 \mathrm{mmHg}$ or a mean DBP $\geqslant 90 \mathrm{mmHg}$ (that is, stage 1 hypertension and above $)^{13}$ were invited for follow up. Although MBP is the best predictor of morbidity and mortality, for the purposes of actually identifying those with hypertension, the World Health Organization definition was used. A letter was delivered to each subject either by post or by hand at the first meeting. All subjects invited for follow up had a fixed abode. Those subjects that did not re-attend were reminded by telephone and invited back at a time convenient to them. When a subject attended follow up they were seen promptly, and three seated right arm measurements were made after 5 minutes rest with 2 minutes between each measurement. A pain score using the same visual scale was obtained. If the SBP was $\geqslant 140 \mathrm{mmHg}$ or the DBP was $\geqslant 90 \mathrm{mmHg}$ at the third reading the patients were asked to give consent to their GP being informed. When subjects were not GP registered, a letter for a GP was sent to their home address and they were urged to register.

\section{STATISTICAL METHODS}

\section{Sample size calculation}

A pilot study of 41 subjects showed mean SBP to be $131 \mathrm{mmHg}$ and mean DBP to be $82 \mathrm{mmHg}$ with SD of $10.8 \mathrm{mmHg}$. Hence, to have a $90 \%$ chance of seeing the mean SBP fall by $10 \mathrm{~mm}$ from $131 \mathrm{mmHg}$ at baseline to $121 \mathrm{mmHg}$ at recall, or having the DBP fall by $5 \mathrm{mmHg}$ to $77 \mathrm{mmHg}$ at recall, and declaring this significant using a two tailed test $(\alpha=0.05), 42$ subjects would be required at follow up. The distribution of data was tested for normality and a two tailed $t$ test used to assess the difference between means. A p value of 0.05 was taken to be significant.

\section{RESULTS}

In total, 765 subjects were seen in total during the 6 week study, of whom $213(27.8 \%)$ were hypertensive. After excluding those receiving antihypertensive treatment or with a history of the condition and those who lived abroad or who were temporarily in the UK, $126(16.5 \%)$ subjects were invited for follow up, of whom 51 (40\%) actually attended their appointments. The average time lag between presentation and follow up was 12.4 days (range 5 to 23). The number of hypertensive subjects at follow up was 39, (5\% of the sample population), and all subjects attended the ED purely for the purposes of follow up. Fig l shows an overview of the results.

\section{Follow up}

Of the 51 subjects who attended the ED purely for the purposes of follow up over a 30 day period, 45 (88.2\%) had blood pressure in the high to normal category or above at the third reading. The total number found to be stage 1 hypertensive or higher was 39 (76.5\%), of whom 17 were not GP registered. Six subjects fell into the normal and optimal categories $(11.8 \%)$. A paired $t$ test was performed, comparing the mean MBP at presentation with the third MBP reading obtained at follow up of each of the 51 subjects who attended follow up. There was a significant difference in MBP at follow up compared with presentation $(\mathrm{p}<0.001)$. As two subjects did not give their consent to us informing their GP and 17 subjects were not GP registered, 20 letters were sent to GPs informing them of our findings, and 17 letters went to the home addresses of those patients who were not GP registered.

\section{Sex specific follow up}

In total, $76 \%$ of men and $68 \%$ of women remained stage 1 hypertensive or above at follow up.

\section{The effects of pain on blood pressure}

Those subjects who presented with a 10/10 pain score had a mean MBP $8.4 \mathrm{mmHg}$ higher than the mean for all other PS $(\mathrm{p}<0.1)$ (fig 2). By comparing the 13 subjects who presented with severe pain $(>5 / 10)$ who were subsequently seen at follow up with pain $<1 / 10$, we could see the effects of severe pain on blood pressure (fig 3 ).

\section{DISCUSSION}

This study showed that $5 \%$ of ED attendees (triage category 3-5) had hypertension that was sustained at follow up. In an ED with 53940 patients attending per year, this represents at total of at least 2697 patients annually. Although the mean MBP was significantly lower at follow up than at presentation, 39 subjects $(76.4 \%)$ remained hypertensive. Of the $40 \%$ attending follow up, the results equate to a prevalence of previously undiagnosed hypertension, confirmed by follow 


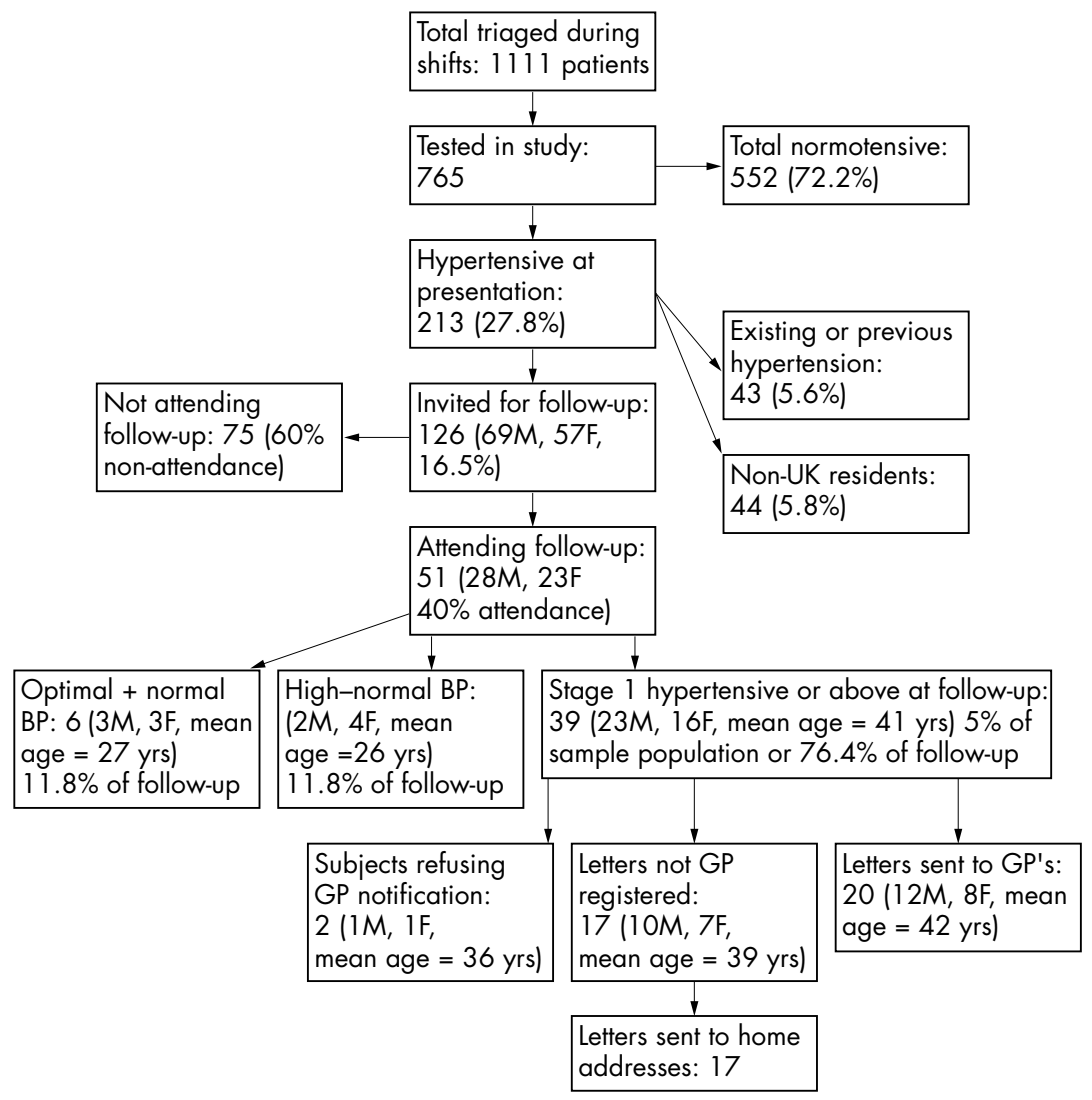

Figure 1 Summary of study procedure and outcomes.

up, of $5 \%$. The follow up rate could have been improved if a longer period had been allowed.

It is unclear whether those attending follow up were representative of the sample population with raised blood pressure on presentation. Those who attended their follow up appointment might have been more likely to want to change their health behaviour and make positive changes to their lifestyle to reduce their overall cardiovascular risk. Follow up contributions by these subjects may underestimate the prevalence of a condition such as hypertension. However, those who did not re-attend, despite phone calls encouraging them to do so, may be more likely to avoid such health promotion efforts. It is not known whether those subjects found to be hypertensive at follow up were commenced on successful treatment. Further study could follow subjects referred to primary care for assessment and treatment, allowing the usefulness of this case finding exercise to be evaluated.

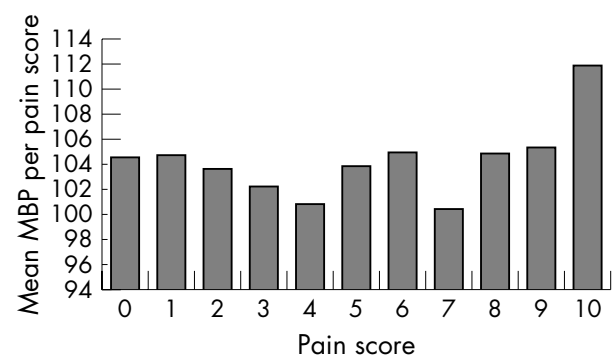

Figure 2 Relationship between the average MBP for each pain score.
In any scenario involving blood pressure measurement, the lability of the haemodynamic system poses challenges. It is hoped that the strict BP measurement protocol employed in this study alleviated the effects of "white coat" hypertension. SBP was particularly labile and we hoped that the third reading at follow up permitted a suitable regression of a subject's MBP to the mean.

Of 13 hypertensive subjects presenting to the ED with pain rated $>5 / 10,62 \%$ remained hypertensive at follow up, even though the mean PS was then $<1 / 10$. The inherent subjectivity of the visual analogue scale cannot be overlooked. ${ }^{12}$ The effects of over the counter analgesics, pain produced only on

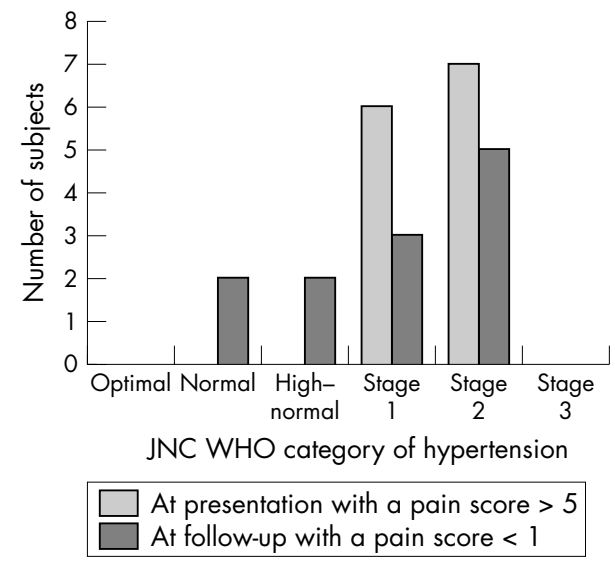

Figure 3 Subjects in severe pain $(>5 / 10)$ who had $<1 / 10$ pain at follow up. 


\section{WHAT IS ALREADY KNOWN ON THIS TOPIC}

- Hypertension is a major risk factor for cardiovascular morbidity and mortality.

- The urban poor have little access to health care delivery sites other than an ED and regard it as their regular, and frequently their only, source of care.

- The government aims to reduce coronary heart disease and stroke mortality by at least $40 \%$ in people $<75$ years of age by the year 2010.

- Hypertension can be effectively controlled by lifestyle changes and pharmacological intervention.

\section{WHAT THIS STUDY ADDS}

- Detection of hypertension is feasible in the ED.

- $5 \%$ of ED attendees remained hypertensive at follow up.

- The ED could enhance disease detection in this hard to reach population.

- Caution is advised when diagnosing hypertension in those ED patients with acutely painful presenting complaints.

- Hypertension in the ED patient population should not automatically be put down to a painful presenting complaint, and repeat measurement should be performed before discharge.

physical movement, and chronicity further blur the interpretation of pain at the time of presentation. Although not statistically significant, the $8.4 \mathrm{mmHg}$ rise in MBP of those subjects experiencing "maximum" pain is interesting.

The UK government aims to reduce coronary heart disease and stroke mortality by at least $40 \%$ in people $<75$ years of age by the year 2010. ${ }^{14}$ A "new approach" is proposed, highlighting the three way partnership between individuals, communities, and government. Tackling the mortality and morbidity experienced by those in lower socioeconomic groups is central to this approach, and demands radical and persistent attention. The ED provides an invaluable health resource and in some urban areas, a third of all attendees view it as their main source of primary care (data from patient records system St Mary's Hospital, London, accessed March 2003).

This same sector of society suffers the highest rates of cardiovascular morbidity and mortality. ${ }^{15}$ For these groups, health interventions can only be made through an outreach programme or whilst attending the ED. By proactively assisting in the GP registration process, and promoting health awareness during attendance, the ED could enhance disease detection in this hard to reach population.

\section{THIS WEEK IN THE EMJ}

Hypertension is well known as a major risk factor for cardiovascular and cerebrovascular morbidity and mortality. However, in ethnic minority communities; $32.5 \%$ of hypertensive individuals still remain undiagnosed despite the increased emphasis on detection. ${ }^{16}$ The National Service Framework aims to reduce coronary heart disease and stroke mortality by at least $40 \%$ in people $<75$ years of age by the year 2010. The GP is often the person given the task of diagnosing hypertension in the community. This study by Fleming et al examines the feasibility of detecting hypertension in the ED, which is often used by those who never attend a GP. It found that $5 \%$ of ED patients had undiagnosed hypertension. This is another way of tackling the burden of cardiovascular illness.

\section{ACKNOWLEDGEMENTS}

We thank Professor K Jamrozik for statistical and methodological advice.

\section{Authors' affiliations}

J Fleming, C Meredith, J Henry, Academic Department of Accident and Emergency Medicine, Imperial College, St Mary's Hospital, London, UK Competing interests: there are no competing interests.

Ethics approval was granted by the St Mary's local research ethics committee.

\section{REFERENCES}

1 Castelli WP. Epidemiology of coronary heart disease: the Framingham Study. Am J Med 1984:76:4-12

2 Uemura K, Piza Z. Trends in cardiovascular disease mortality in industrialized countries since 1950. World Health Stat Q 1988;41:155-78.

3 Kannel WB. Prevalence and implications of uncontrolled systolic hypertension. Drugs Aging 2003;20:277-86.

4 Lewington S, Clarke R, Qizilbash N, et al. Age-specific relevance of usual blood pressure to vascular mortality: a meta-analysis of individual data for one million adults in 61 prospective studies. Lancet 2002;360:1903-13.

5 Heart Forum. Looking to the future: making coronary heart disease an epidemic of the past. London: The Stationery Office, 1998.

6 Gibson G. EMS: A facet of ambulatory care. Hospitals 1973;5:51-6.

7 Department of Health. Service framework for coronary heart disease. London: DoH, 2002.

8 Chiang WK, Jamshahi B. Asymptomatic hypertension in the ED. Am J Emerg Med 1998;16:701-704.

9 Rose G. Standardisation of observers in blood pressure measurement. Lancet 1965;1:673-4.

10 Lehmann KG, Gelman JA, Weber MA, et al. Comparative accuracy of three automated techniques in the noninvasive estimation of central blood pressure in men. Am J Cardiol, 1998 Apr 15, 81:1004-12.

11 Davis JW, Davis IC, Bennink LD, et al. Are automated blood pressure measurements accurate in trauma patients? J Trauma 2003;55:860-3.

12 Bijur P, Latimer C, Gallagher E. Validation of a verbally administered numerical rating scale of acute pain for use in the emergency department. Acad Emerg Med 2003;10:390-2.

13 Joint National Committee on Prevention, Detection Evaluation and Treatment of High Blood Pressure (JNC-V1). Sixth report. Arch Intern Med 1997; 157:2413-46.

14 Department of Health. DoH website. /www.doh.gov.uk/ohn.htm. Accessed December, 2003

15 Blakely T, Woodward A, Pearce N, et al. Socio-economic factors and mortality among 25-64 year olds followed from 1991 to 1994: the New Zealand Census-Mortality Study. N Z Med J 2002;115:93-7.

16 Memon M, Abbas F, Memon B. Assessing CHD and hypertension in minority ethnic communities. Nurs Times 2003;9916:26-7. 
COMMENTARY

\section{Should emergency departments really be screening for hypertension?}

\section{J Lee}

$\mathrm{F}$ leming et al, ${ }^{1}$ in this issue of the $E M J$, present further evidence that hypertension identified in the emergency department (ED) should not simply be dismissed as secondary to pain, anxiety or "white coat" effect. Their finding that $28 \%$ of patients with "minor injuries" were hypertensive should not surprise, as the British Society for Hypertension reports that $42 \%$ of $35-64$ year olds in the UK have hypertension. ${ }^{2}$ Clearly, we can screen for hypertension in the ED but should we do so?

Few would disagree that tight control of blood pressure across the UK population would prevent a significant number of deaths from myocardial infarction and stroke. Individually, however, hypertension is controlled in only a third of treated patients. ${ }^{3}$ In this study, only $40 \%$ of patients with identified hypertension returned for follow up and of these it is not known how many subsequently registered with a general practitioner (GP) and complied with medication. Fleming et al argue that for many patients (particularly in inner cities) the ED is their primary contact with health services and that without screening their hypertension would otherwise remain unidentified. Patients with no fixed abode would be expected to fall into this category, but hypertension was not identified in anyone from this sub-group.

Screening is not new to the ED. In fact, we have a duty to screen for conditions that may be presented to the ED rather than the GP, particularly where identification may immediately prevent further injury, such as in cases of intimate partner violence and nonaccidental injury. A recent study from the authors' own centre ${ }^{4}$ showed that screening and counselling of patients presenting to the ED with conditions associated with alcohol misuse resulted in a clear reduction in use of the ED by the same patients in the following 12 months. Both of the above are examples of targeted screening, which is more efficient and cost effective than universal screening but inevitably misses more cases. Although Fleming et al describe their screening as targeted, it is in essence universal screening of all patients assigned a triage category of 3-5. EDs do not have a duty, or the resources, to universally screen for asymptomatic, chronic conditions. Unless such screening can demonstrate an immediate health benefit or reduction in ED attendance, our focus should instead remain on improving our performance of core roles. This study, understandably, cannot demonstrate either benefit.

If money is made available to EDs for targeting hypertension it may be better used to assist patients who are not currently registered with a GP to do so. GPs, for their part, can be relied upon to monitor blood pressure, because, as of April 2004, part of their income has been related to the control of their patients' hypertension. Furthermore, registration with a GP would allow health care needs other than blood pressure control to be addressed and may reduce future visits to the ED.

Fleming et al have demonstrated that screening for hypertension in the ED is feasible but will not convince all that screening for it in the ED is an effective or efficient use of resources. GP "refer-

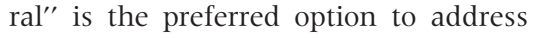
hypertension in hard to target groups.

Emerg Med J 2005;22:640.

doi: 10.1136/emj.2004.018747

Correspondence to: Mr Jason Lee, Accident and Emergency, St James's University Hospital, Leeds LS9 7TF, UK; docjasonlee@hotmail.com

Accepted for publication 17 January 2005

Competing interests: none declared

\section{REFERENCES}

1 Fleming J, Meredith C, Henry J. Detection of hypertension in the emergency department. Emerg Med J 2005;22:636-9

2 Williams B, Poulter NR, Brown MJ, et al. Guidelines for the management of hypertension: report of the fourth working party of the British Hypertension Society - BHS IV. J Hum Hypertens 2004; 18:139-85.

3 Primatesta P, Poulter NR. Hypertension management and control among English adults aged 65 years and older in 2000 and 2001. $J$ Hypertens 2004;22:1093-8.

4 Crawford MJ, Patton R, Touquet R, et al. Screening and referral for brief intervention of alcohol-misusing patients in an emergency department: a pragmatic randomised controlled trial. Lancet 2004;364:1334-9. 